\title{
Methods, Challenges and Future Directions of Radiogenomics
}

\author{
Lehui $\mathrm{Du}^{1}$, Hui Zheng ${ }^{2 *}$ and Baolin Qu ${ }^{1 *}$ \\ ${ }^{1}$ Department of Radiation Oncology, China \\ ${ }^{2}$ Tianjia Genomes Tech CO., LTD., China
}

ISSN: 2637-773X

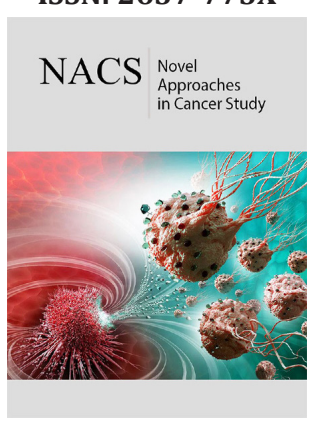

*Corresponding author: Baolin Qu, Department of Radiation Oncology, Chinese PLA General Hospital, China and Hui Zheng, Tianjia Genomes Tech CO., LTD., China

Submission: 眥 April 13, 2020

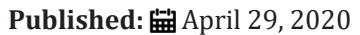

Volume 4 - Issue 4

How to cite this article: Lehui $\mathrm{Du}$ Hui Zheng, Baolin Qu. Methods, Challenges and Future Directions of Radiogenomics. Nov Appro in Can Study. 4(4). NACS.000592. 2020 DOI: 10.31031/NACS.2020.04.000592

Copyright@ Hui Zheng and Baolin Qu. This article is distributed under the terms of the Creative Commons Attribution 4.0 International License, which permits unrestricted use and redistribution provided that the original author and source are credited.

\section{Abstract}

Tissue response to the radiation is a complex pathophysiological process and is an inherited polygenic trait. Aim of the Radiogenomics studies is to discover related genetic variants that confer tumor or nontumor tissue radio sensitivity as the target of radio-sensitizing and/or radio-protective agents and to identify specific genetic markers for prognosis or risk prediction. The methods for radiogenomics studies include candidate gene approaches, genome-wide association studies, Next-Generation Sequencing (NGS), epigenetic study, and other methods. The future direction of radiogenomics should be the development of the polygenic risk scores that are incorporated into end point-specific clinical models/nomograms.

\section{Introduction}

As one of major cancer treatments, radiotherapy benefits more than half of cancer patients [1]. After many years of development, just like other cancer therapies the radiotherapy also evolved into the era of precision medicine, which addresses each therapy should be tailored to the personal characteristics. One important aspect of precision radiotherapy is genomic studies, often referred as radiogenomics in the field. There two main goals for radiogenomics. One is to discover related genetic variants that confer tumor or non-tumor tissue radiosensitivity as the target of radio-sensitizing and/or radio-protective agents. Another goal is to identify specific genetic markers for prognosis or risk prediction. Tissue response to the radiation is a complex pathophysiological process and is an inherited polygenic trait that accounts for up to $80 \%$ of the differences in radiosensitivity [2]. In vitro studies have also estimated a range from 58 to $82 \%$ of the heritability of cellular radiosensitivity [3]. Radiosensitivity or radiotoxicity is affected by multiple genes and/or biological pathways that include a few rare mutations that confer large effects and multiple common variants with small effects. This review is to summarize major research approaches and advances in radiogenomics.

\section{Candidate Gene Approaches}

In general, candidate genes are those genes that known or thought to be involved in the pathway of radiosensitivity or radiotoxicity. The early radiogenomics studies mainly used candidate gene approaches. For quite a long period radio-oncologists already noticed that substantial genetic variationscould alterthe severity of tissue reactions to radiotherapy. Back to the 1960s, it has been reported that early Ataxia-telangiectasia patients suffered severe acute radiotoxicity, which indeed is the ATM mutation accounting for the symptom [4]. Until recent still ATM mutations were discovered associated with excellent radiation response [5]. ATM probably is one of the earliest genes known and therefore used as a candidate gene for radiogenomics. Such kinds of candidate genes usually are genes that involved in DNA repair (ATM, BRCA1, CHEK2), anti-oxidation (SOD2), apoptosis (TP53), and cytokine signaling (TBFB1), etc. The major advantage of candidate gene studies is low cost. With focusing on limited specific variants, one can collect more samples and therefore results with more statistical power. While the drawback of this method is the requirement of prior knowledge of gene function. As mentioned before the radiosensitivity or radiotoxicity is a combined effect of different numbers of rare variants and common variants. The total number of those variants can be from tens to hundreds and many of them could be with unknown function. This means the candidate gene approach could miss many genes involved in the radiosensitivity. Despite the disadvantages, the International Radiogenomics Consortium (RGC) has reported several 
successful works using candidate gene studies, which discovered several SNPs associated with radiotoxicity in near or in candidate genes such as TNF, XRCC, and ATM [6-8]. Now a day with the cost decreasing of other methods, the candidate gene method is no longer competitive yet stills a useful method especially for small institutes with a limited budget. Caution should be paid when using this method since some prior knowledge could be misleading such as in TGF- $\beta$ study [9-12] and early radiogenomics studies using this method failed to account for multiple testing [13].

\section{Genome-Wide Association Studies}

With the advances in DNA chip, the genome-wide association studies (GWAS) is getting more popular than the candidate gene studies. One obvious advantage of GWAS is to allow researchers to study all SNPs without prior knowledge which means variants with unknown functions can be discovered and the method is suitable for exploratory researches. The first radiogenomics GWAS identified rs2268363 (unadjusted $p=5.46 \times 10-8$, Bonferroni $p=0.028$ ) in the follicle-stimulating hormone receptor (FSHR) gene associated with erectile dysfunction (ED) among a cohort of 79 African American prostate cancer patients treated with external beam radiotherapy [14]. Later several other loci in the two-stage GWAS were also identified by the same group (Rosenstein group) among post radiotherapeutic prostate cancer patients associated with urinary toxicity or rectal bleeding $[15,16]$. Many modern radiogenomics GWASs are often designed with two or multi-stages with cohorts of a large population $(>1000)$, the first stage for discovery and the second stage for validation, UK RAPPER study, for example [17]. Such kind of design can provide solid evidence for the variants and phenotype association. GWAS can identify common variants with moderate or small effects. The biggest problem for GWAS is that most identified loci usually located in the noncoding region, which makes it difficult to explain the underlying mechanism although it's not necessary for clinical utilization. Another flaw for GWAS is most GWAS studies will exclude rare variants, which is essential for radiogenomics.

\section{Next-Generation Sequencing (NGS) Approach}

Next-Generation Sequencing, also called high-throughput sequencing, massively parallel sequencing, or deep sequencing, can generate a huge amount of information within one run. Comparing to other methods, NGS has many advantages. The first advantage is the cost for each variant is very low. Now a whole human genome can be sequenced for around a thousand dollars, which equals about only thirty cents per million bases. The second advantage is NGS can be applied at different scales: either focusing on few or multiple specific genes, or the whole exome, or the whole genomes. The third is the same set of NGS data can also be used for copy number alterations (CNA) analysis, which is an important yet to be explored area for radiogenomics. The fourth one is NGS can also be used for epigenetic research for radiogenomics, another important yet to be explored area for radiogenomics. The fifth advantage is the small Indels, which is extremely important for some specific genes such as TP53, difficult to be found with other methods, can be easily discovered by NGS. There so many successful applications of NGS in radiogenomics [18] and here we will give only one typical example addressing one specific feature of NGS. In a study of concurrent chemoradiation of squamous cell carcinoma of the head and neck (HNSCC) patients where the NGS panel was applied, the null functional (nonsense, frameshift, truncation, or complete loss of expression) TP53 was found distinct from the missenses mutations as far as the survival concerned [19]. This study is a typical example illustrating varieties of complex mutations that can be detected by NGS simultaneously. One thing that should be noticed is that NGS generates huge amounts of data and analysis is a time-consuming process often requires powerful computing and bioinformatic resources.

\section{Epigenetic Study and Other Methods}

Epigenetic modifications include DNA methylation and histone modifications (methylation and acetylation). Both will cause gene expression profile change, which could result in the variation of sensitivity or resistance of tumor or non-tumor tissues to radiation. At least it has been shown in vitro the DNA methylation changes were associated with radioresistance in a human laryngeal squamous cell carcinoma cell line [20]. As for the radiogenomics studies only very few literatures regarding epigenetics were published so far and it is a direction with many prospects. Another direction of radiogenomics is gene expression profiling. A typical example is the work did by Torres-Roca group which used a gene-expression-based radiation-sensitivity index (GARD)to independently predicted the clinical outcome of different cancers [21].

\section{Challenges and Future Directions}

To date, continuous advances in new techniques brought new challenges to radiogenomics studies. The one emerging radiotherapy is the increasing use of charged particle therapy including protons and carbon ions. Different types of radiation will have different radiotoxicity. How different those radiotoxicities are and whether previous knowledge on the radiogenomics can be applied to these new techniques? Even with the traditional type of radiation, methods like Oligo fractionation, which intents to improving tumor cell killing, delivers much higher radiation doses in fewer fractions than standard fractionation, and the question is whether radiotoxicity risk differs from standard fractionation. The biological effect of radiation includes but is not restricted to the generation of DNA damage. Many other pathways such as cell death, immunological response, hypoxia metabolism, etc. are also involved in the radiation response [22,23]. How the genetic variants or gene expression profiles are linked to these biological pathways is another direction of radiogenomics. So far, most of the previous radiogenomics studies have focused on single or few biomarkers as a predictor of radiosensitivity and the polygenic nature of radiosensitivity made none of those biomarkers are currently used in the clinical daily practice. The future direction of radiogenomics should be the development of the polygenic risk scores that are incorporated into end point-specific clinical models/nomograms[24]. 


\section{Acknowledgement}

This work is supported by National Center for Research Center of Geriatric Diseases (NCRCG-PLAGH-2018003).

\section{References}

1. Delaney G, Jacob S, Featherstone C, Barton M (2005) The role of radiotherapy in cancer treatment: Estimating optimal utilization from a review of evidence-based clinical guidelines. Cancer 104(6): 1129-1137.

2. Turesson I, Nyman J, Holmberg E, Odén A (1996) Prognostic factors for acute and late skin reactions in radiotherapy patients. Int J Radiat Oncol Biol Phys 36(5): 1065-1075.

3. Kerns SL, Chuang KH, Hall W, Werner Z, Chen Y, et al. (2018) Radiation biology and oncology in the genomic era. Br J Radiol 91(1091): 20170949.

4. Gotoff SP, Amirmokri E, Liebner EJ (1967) Ataxia telangiectasia Neoplasia, untoward response to $\mathrm{x}$-irradiation, and tuberous sclerosis. Am J Dis Child 114(6): 617-625.

5. Ma J, Setton J, Morris L, Albornoz PB, Barker C, et al. (2017) Genomic analysis of exceptional responders to radiotherapy reveals somatic mutations in ATM. Oncotarget 8(6):10312-10323.

6. Talbot CJ, Tanteles GA, Barnett GC, Burnet NG, Chang-Claude J, et al. (2012) A replicated association between polymorphisms near TNF alpha and risk for adverse reactions to radiotherapy. Br J Cancer 107(4):748753.

7. Seibold P, Behrens S, Schmezer P, Helmbold I, Barnett G,et al. (2015) XRCC1 polymorphism associated with late toxicity after radiation therapy in breast cancer patients. Int J Radiat Oncol Biol Phys 92(5):1084-1092.

8. Andreassen CN, Rosenstein BS, Kerns SL, Ostrer H, De Ruysscher D, et al. (2016) Individual patient data meta-analysis shows a significant association between the ATM rs1801516 SNP and toxicity after radiotherapy in 5456 breast and prostate cancer patients. Radiother Oncol 121(3): 431-439.

9. Andreassen CN, Alsner J, Overgaard J, Herskind C, Haviland J, et al. (2005) TGFB1 polymorphisms are associated with risk of late normal tissue complications in the breast after radiotherapy for early breast cancer. Radiother Oncol 75(1): 18-21.

10. Andreassen CN, Alsner J, Overgaard M, Sørensen FB, Overgaard J, et al. (2006) Risk of radiation-induced subcutaneous fibrosis in relation to single nucleotide polymorphisms in TGFB1, SOD2, XRCC1, XRCC3, APEX and ATM--a study based on DNA from formalin fixed paraffin embedded tissue samples. Int J Radiat Biol 82(8): 577-586.

11. Barnett GC, Coles CE, Elliott RM, Baynes C, Luccarini C, et al. (2012) Independent validation of genes and polymorphisms reported to be associated with radiation toxicity: A prospective analysis study. Lancet Oncol 13(1): 65-77.

12. Alsner J, Andreassen CN, Overgaard J (2008) Genetic markers for prediction of normal tissue toxicity after radiotherapy. Semin Radiat Oncol 18(2): 126-135.
13. Brothwell MRS, West CM, Dunning AM, Burnet NG, Barnett GC, et al. (2019) Radiogenomics in the era of advanced radiotherapy. Clin Oncol (R Coll Radiol) 31(5): 319-325.

14. Kerns SL, Ostrer H, Stock R, Li W, Moore J, et al. (2010) Genome-wide association study to identify single nucleotide polymorphisms (SNPs) associated with the development of erectile dysfunction in AfricanAmerican men after radiotherapy for prostate cancer. Int J Radiat Oncol Biol Phys 78(5):1292-1300.

15. Kerns SL, Stock RG, Stone NN, Blacksburg SR, Rath L, et al. (2013) Genome-wide association study identifies a region on chromosome $11 q 14.3$ associated with late rectal bleeding following radiation therapy for prostate cancer. Radiother Oncol 107(3): 372-376.

16. Kerns SL, Stone NN, Stock RG, Rath L, Ostrer H, et al. (2013) A 2 -stage genome-wide association study to identify single nucleotide polymorphisms associated with development of urinary symptoms after radiotherapy for prostate cancer. J Urol 190(1):102-108.

17. Barnett GC, Thompson D, Fachal L, Kerns S, Talbot C, et al. (2014) A genome wide association study (GWAS) providing evidence of an association between common genetic variants and late radiotherapy toxicity. Radiother Oncol 111(2): 178-185.

18. Bibault JE, Tinhofer I (2017) The role of Next-generation sequencing in tumoral radiosensitivity prediction. Clin Transl Radiat Oncol 3:16-20.

19. Tinhofer I, Stenzinger A, Eder T, Konschak R, Niehr F, et al. (2016) Targeted next-generation sequencing identifies molecular subgroups in squamous cell carcinoma of the head and neck with distinct outcome after concurrent chemoradiation. Ann Oncol 27(12): 2262-2268.

20. Kim JS, Kim SY, Lee M, Kim SH, Kim SM, et al. (2015) Radioresistance in a human laryngeal squamous cell carcinoma cell line is associated with DNA methylation changes and topoisomerase II alpha. Cancer Biol Ther 16(4): 558-566.

21. Scott JG, Berglund A, Schell MJ, Mihaylov I, Fulp WJ, et al. (2017) A genome-based model for adjusting radiotherapy dose (GARD): A retrospective, cohort-based study. Lancet Oncol 18(2): 202-211.

22. Bibault JE, Fumagalli I, Ferte C, Chargari C, Soria JC, et al. (2013) Personalized radiation therapy and biomarker-driven treatment strategies: A systematic review. Cancer Metastasis Rev 32(3-4): 479492 .

23. Liauw SL, Connell PP, Weichselbaum RR (2013) New paradigms and future challenges in radiation oncology: An update of biological targets and technology. Sci Transl Med 5(173): 173sr2.

24. Du L, Ma N, Dai X, Yu W, Huang X, et al. (2020) Precise prediction of the radiation pneumonitis in lung cancer: an explorative preliminary mathematical model using genotype information. J Cancer 11(8): 23292338. 